\title{
Low-Energy Irradiation Damage in Single-Wall Carbon Nanotubes
}

\author{
Satoru Suzuki \\ NTT Basic Research Laboratories, NTT Corporation, 3-1, Morinosato Wakamiya, \\ Atsugi, Kanagawa \\ Japan
}

\section{Introduction}

Single-wall carbon nanotubes (SWCNTs) are one of the most promising materials for future nano-electronics, because of their unique quasi-one-dimensional structures and excellent electric and mechanical properties. They also have very high chemical stability, owing to their robust sp2-bonding carbon network (graphene) with no dangling bonds. Because of the structural robustness, low-energy (typically $10 \mathrm{eV}-20 \mathrm{keV}$ ) electron and photon irradiation in a vacuum had been generally assumed not to cause damage to SWCNTs when the energy is smaller than the knock-on threshold. In fact, analytical tools that use low-energy electrons or photons, such as scanning electron microscopy (SEM), had been commonly used for characterization of SWCNTs without serious concerns.

In 2004, however, we reported that electron irradiation in a SEM caused severe damage (low-energy irradiation damage) in SWCNTs produced by both thermal chemical vapor deposition and laser ablation methods (Suzuki et al., 2004b). Other techniques using lowenergy electrons and vacuum-ultraviolet (VUV) light or soft x-rays (especially highbrilliance synchrotron radiation light), such as low-energy electron microscopy (LEEM) and photoemission spectroscopy, also inevitably damage SWCNTs. Therefore, paying attention to the low-energy irradiation damage is practically important for those who study SWCNTs. For example, when we measure the Raman and photoluminescence (PL) spectra and electric properties and take SEM images of the same SWCNTs, the SEM observations should be done last. Doing the high-resolution SEM observation first would inevitably cause severe damage and tremendously affects the following measurements.

The low-energy irradiation damage and its defect characteristics are also physically interesting. In this chapter, we will review the physical and chemical property changes induced by the damage, and the defect properties, which are significantly different from those of other types of damage. We will examine the defect-induced metal-semiconductor transition of the room-temperature electric properties and discuss its mechanism. We will also summarize other types of damage, which are often confused with the low-energy irradiation damage, focusing on the differences between them.

Before continuing to the main text, I must briefly explain how I compare spectra obtained form the same SWCNT sample. In many studies of the physical or chemical treatment of SWCNTs and graphene, spectra are often normalized to the maximum peak height. In 
contrast, when I show irradiation- and annealing-induced changes of Raman and PL spectra, the spectra are never normalized. That is, I obtain the spectra under the same condition to the best of my ability and directly compare the raw spectra. This methodology has been applied in all of our related reports, unless otherwise mentioned. With arbitrary spectral normalization, we would no longer be able to discuss the reversibility of the damage and recovery, which is a very important characteristic of low-energy irradiation damage.

\section{What is low-energy irradiation damage?}

We define low-energy irradiation damage as damage solely caused by irradiation of lowenergy particles, where low-energy means that the energy is much smaller than the threshold energy of knock-on damage. Thus, the mechanism of the damage is completely different from knock-on damage. Moreover, we discriminate low-energy irradiation damage and secondary damage caused by the irradiation, such as damage by radicals. Irradiation by both electrons and photons irradiation was found to damage SWCNTs. However, other particles, such as atoms and ions, or quasi-particles such as plasmons, may also cause the damage.

\section{Property changes caused by low-energy irradiation damage}

\subsection{Raman and PL spectra}

In a Raman spectrum, a SWCNT shows the so-called G band (tangential mode) and disorder-induced D band, which are characteristic of a graphene sheet. The D band is ideally inactive and its appearance is evidence of symmetry breaking. The intensity ratio of the $G$ and $\mathrm{D}$ bands is often utilized as an indicator of the degree of crystallinity. Another very important mode of SWCNTs is the radial breathing mode (RBM), which is often used for diameter evaluation. For a general review of Raman spectroscopy of CNTs, see (Dresselhaus et al., 2005), for example. Like other types of damage, low-energy irradiation damage generally decreases the $\mathrm{G}$ band and RBM intensities (There are some exceptions at the edges of the resonance window, as discussed below) and the G/D intensity ratio and increases the $\mathrm{D}$ band intensity, as shown in Figs. 1(a) and (b). Generally, the decrease of intensity is more prominent for the RBM than for the $G$ band. Considering that the detectable Raman intensity from individual SWCNTs is owing to the resonance enhancement effect, the disappearance of Raman spectra is probably due to a reduction of the resonance enhancement. The initially divergent joint density of states, which is a characteristic of onedimensional systems, would be considerably broadened by the formation of defects. Lowenergy irradiation damage causes almost no broadening or almost no shift of the Raman peaks including the D band (Suzuki et al., 2010), although significant D band broadening due to gas-phase reaction has been observed (Yang et al., 2006. Zhang et al., 2006).

When SWCNTs are moderately damaged (or considerably recover from severe damage), originally hidden non-resonant RBM peaks sometimes appear. At the excitation wavelength of $785 \mathrm{~nm}$, metallic and semiconducting SWCNTs are usually observed at about 150-160 and 200-240 $\mathrm{cm}^{-1}$, respectively. In Fig. 2(a), however, the moderately damaged (partially recovered) SWCNTs show a sharp peak at $182 \mathrm{~cm}^{-1}$ in the off-resonance region. The metallic SWCNTs at $156 \mathrm{~cm}^{-1}$, which were initially not strongly excited in this sample, also became more prominent in the moderately damaged sample. Further damage extinguishes these peaks again, as also shown in the figure. Similar off-resonant RBM peaks are also often 
observed in doped SWCNTs grown from boron- and nitrogen-containing feedstocks, as shown in Fig. 2(b) (Suzuki \& Hibino, 2011). I think that the defects slightly shift the absorption energy or broaden the absorption edge and this makes the originally off-resonant peak resonant. Similarly, complicated behavior of the RBM intensity with increasing damage is observed at the edge of the resonance window (Suzuki \& Kobayashi, 2007a).
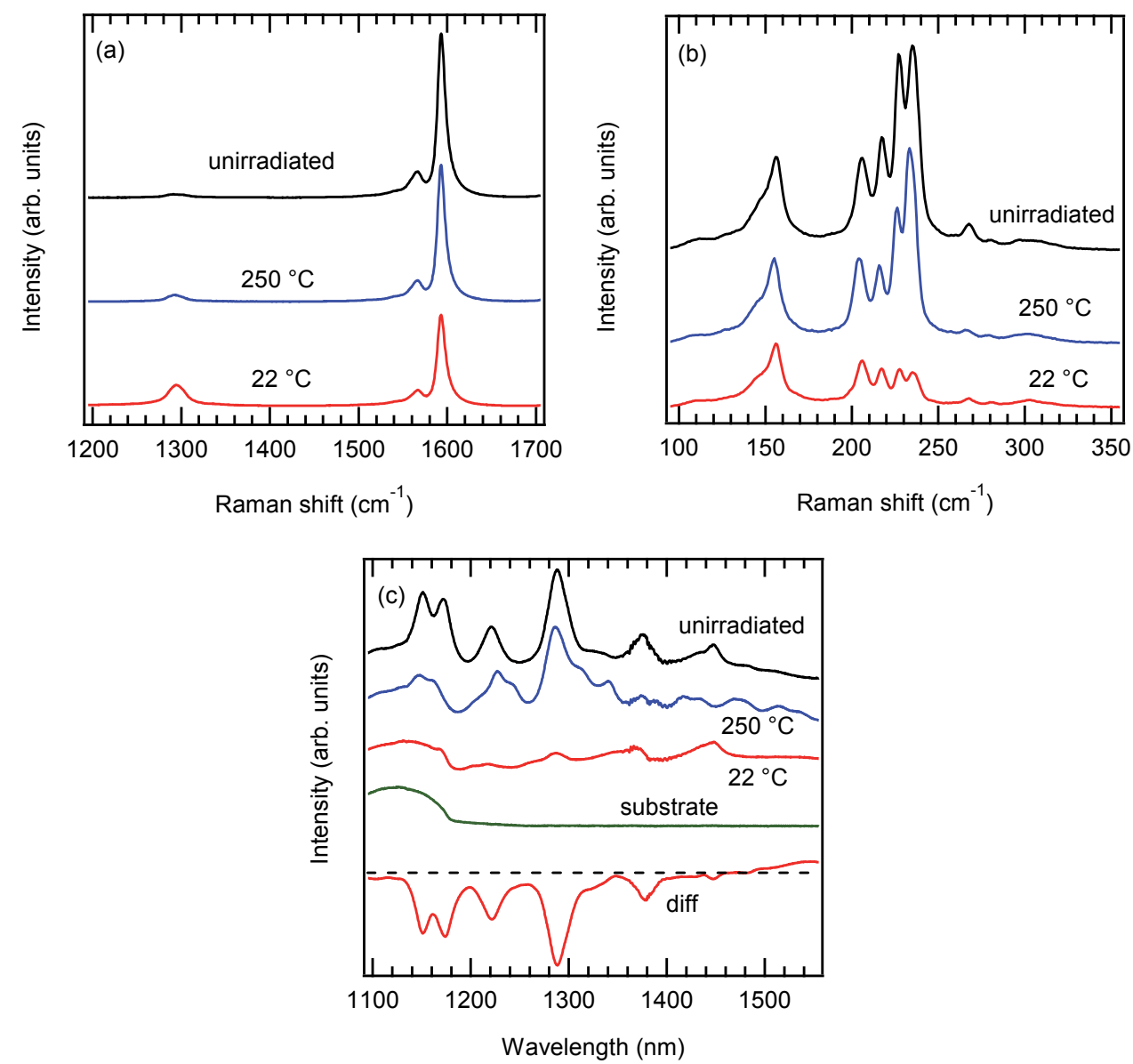

Fig. 1. (a) G and D band, and (b) RBM regions of Raman spectra, and (c) PL spectra of unirradiated SWCNTs and of SWCNTs irradiated at 250 and $22{ }^{\circ} \mathrm{C}$. The irradiated electron energy and dose were $20 \mathrm{keV}$ and $5.7 \times 10^{16} \mathrm{~cm}^{-2}$. The excitation wavelength was $785 \mathrm{~nm}$.

These results mean that the Kataura plot is modified by the defects.

The PL peak intensity of suspended semiconducting SWCNTs is more sensitively decreased than the Raman peak intensity, as shown in Fig. 1(c). In addition, broad spectral intensity newly appears at the longer wavelength side when the extent of the damage is moderate. Severe damage finally extinguishes all spectral intensities in Raman (including the D band (Suzuki et al., 2005a)) and PL spectra (Suzuki \& Kobayashi, 2007b). However, note that, in 
marked contrast to the damage caused by knock-on collisions and by radicals, the lowenergy irradiation damage itself never eliminates a SWCNT.
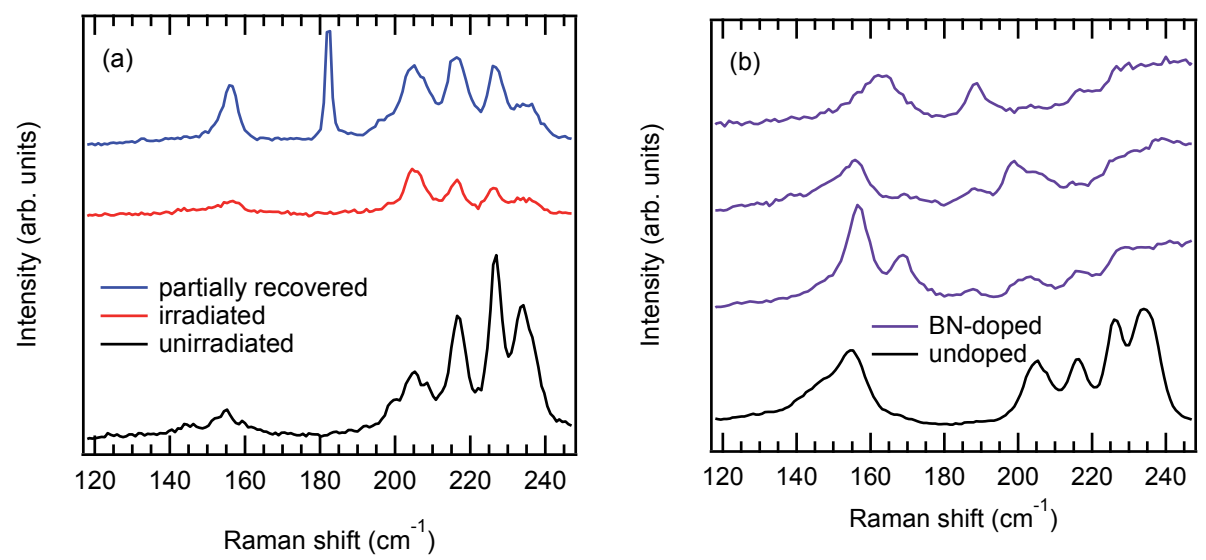

Fig. 2. (a) RBM spectra of unirradiated and electron-irradiated SWCNTs and partially recovered SWCNTs. The electron energy and irradiation dose were $20 \mathrm{keV}$ and $6.3 \times 10^{16} \mathrm{~cm}^{-}$ 2 , respectively. The irradiated and considerably damaged SWCNTs were partially recovered by annealing in Ar atmosphere at $350^{\circ} \mathrm{C}$. The wavenuber range of $160-200 \mathrm{~cm}^{-1}$ is the offresonance region and a peak is rarely observed there, initially. (b) RBM spectra of undoped and BN-doped SWCNTs. The doped SWCNTs also often exhibit peaks in the off-resonance region. The excitation wavelength was $785 \mathrm{~nm}$.
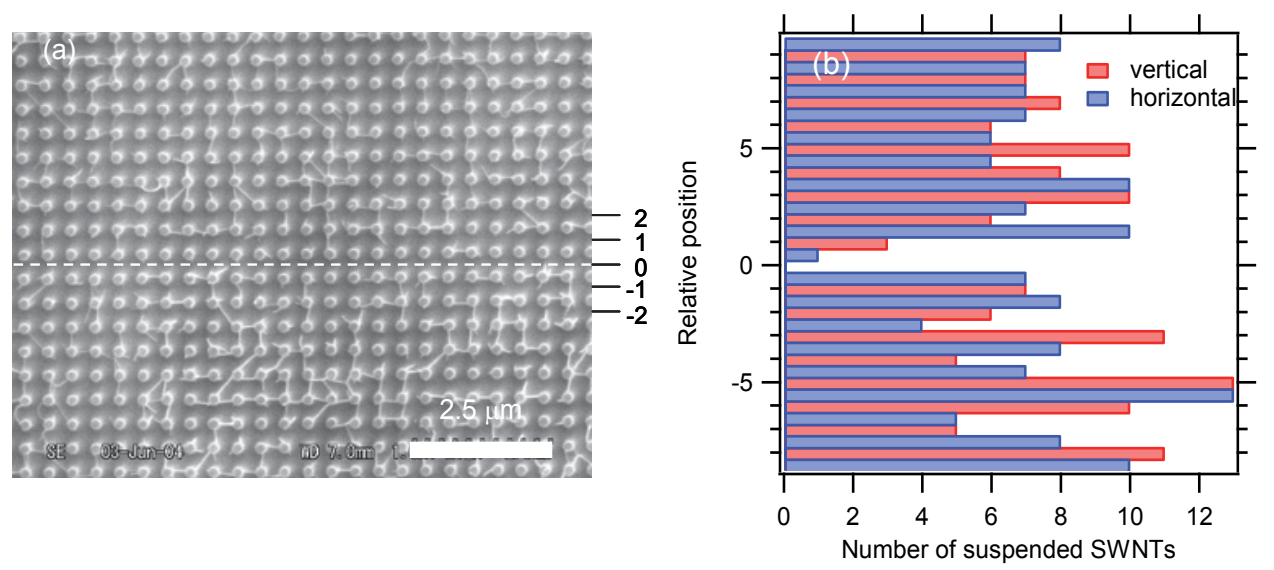

Fig. 3. (a) SEM image of the SWNT sample after eliminating the electron-irradiated SWCNTs. The irradiation was done along the dashed line. The electron energy and the local dose were $1 \mathrm{keV}$, and $1.5 \times 10^{19} \mathrm{~cm}^{-2}$, respectively. Note that the elimination was done by selective combustion in air, not by the irradiation itself. (b) Position dependence of the number of SWCNTs suspended between neighboring pillars in (a). Here, diagonally suspended SWCNTs were neglected. 


\subsection{Chemical stability}

The low-energy irradiation itself does not cut a SWCNT. However, the damage significantly decreases the chemical tolerance of SWCNTs, because the irradiation-induced defects make the sidewall chemically active. Therefore, we can selectively eliminate the irradiated SWCNTs by heating in air, as shown in Fig. 3 (Suzuki et al., 2005a). A part of the SWCNT sample was intensively irradiated in a SEM using the line scan mode along the dashed line. Then, the sample was heated in air at $420{ }^{\circ} \mathrm{C}$ for $30 \mathrm{~m}$. The irradiated SWCNTs were selectively eliminated by combustion due to the reduced chemical tolerance. Note that a irradiation dose that is too high often has an entirely opposite effect, because the irradiationinduced contaminants on the SWCNT surfaces protect the SWCNTs from oxygen. As shown in (b), the irradiation effects almost completely disappear about $600 \mathrm{~nm}$ from the irradiation line. Such a high spatial resolution can be easily obtained using a convergent electron beam. There have been many attempts to functionalize SWCNTs by using other molecules or metal particles. In many cases, defects are intentionally created to functionalize the sidewall, which is originally inert (Yan et al., 2005). Low-energy irradiation damage could also be applied for spatially selective functionalization with electron beam lithography.

\subsection{Electric properties}

The electric properties are much more sensitively changed by low-energy irradiation damage than Raman and PL spectra. Moderate irradiation can convert a metallic field effect transistor (FET) into semiconducting. I will discuss this remarkable phenomenon in sec. 5 . Here, I focus on the intensive irradiation effects I have studied by in-situ electric measurements during electron irradiation in a SEM equipped with piezo-actuated microprobes for electric measurements (Suzuki, 2011). The device used here consists of two SWCNTs (A branch is seen between the electrodes) suspended between the drain and source electrodes (height: $300 \mathrm{~nm}$ ), as shown in Fig. 4(a). The high-magnification SEM image was taken after all experiments had been completed. Otherwise, the conductivity of the SWCNTs would almost vanish. Fig. 4(b) shows the results of in-situ electric measurements during irradiation. The whole SWCNTs were first irradiated by an electron beam using the normal SEM observation mode. The SEM observation gradually decreased the conductivity. Then, at $\sim 42.76 \mathrm{~s}$, they were intensively irradiated by using the line scan mode. This irradiation decreased the conductivity by two orders of magnitude in only a few seconds. As shown in Fig. 4(c), a very abrupt current decrease occurred at least within the initial 44 ms, which is the time resolution of the measurements. The gate voltage characteristics of the device before and after the irradiation are shown in Fig. 4(d). The irradiation decreased the two-probe conductivity by four to five orders of magnitude in the whole gate voltage range. Considering that the initial resistivity of the device would be dominated by the contact resistance between the SWNTs and electrodes, the intrinsic conductivity decrease would be much larger. Thus, intensive irradiation finally makes a SWCNT almost insulating. Similar results had been observed in previous works by another group (Marquardt et al., 2008. and Vijayaraghavan et al., 2010) and in our early work (Suzuki and Kobayashi., 2005), in which conventional on-substrate SWCNT devices were used. Here, I would like to get remind the readers again that even intensive irradiation does not cut a SWCNT. In fact, a SWCNT can be completely recovered by annealing, as shown later in sec. 4.2 . 

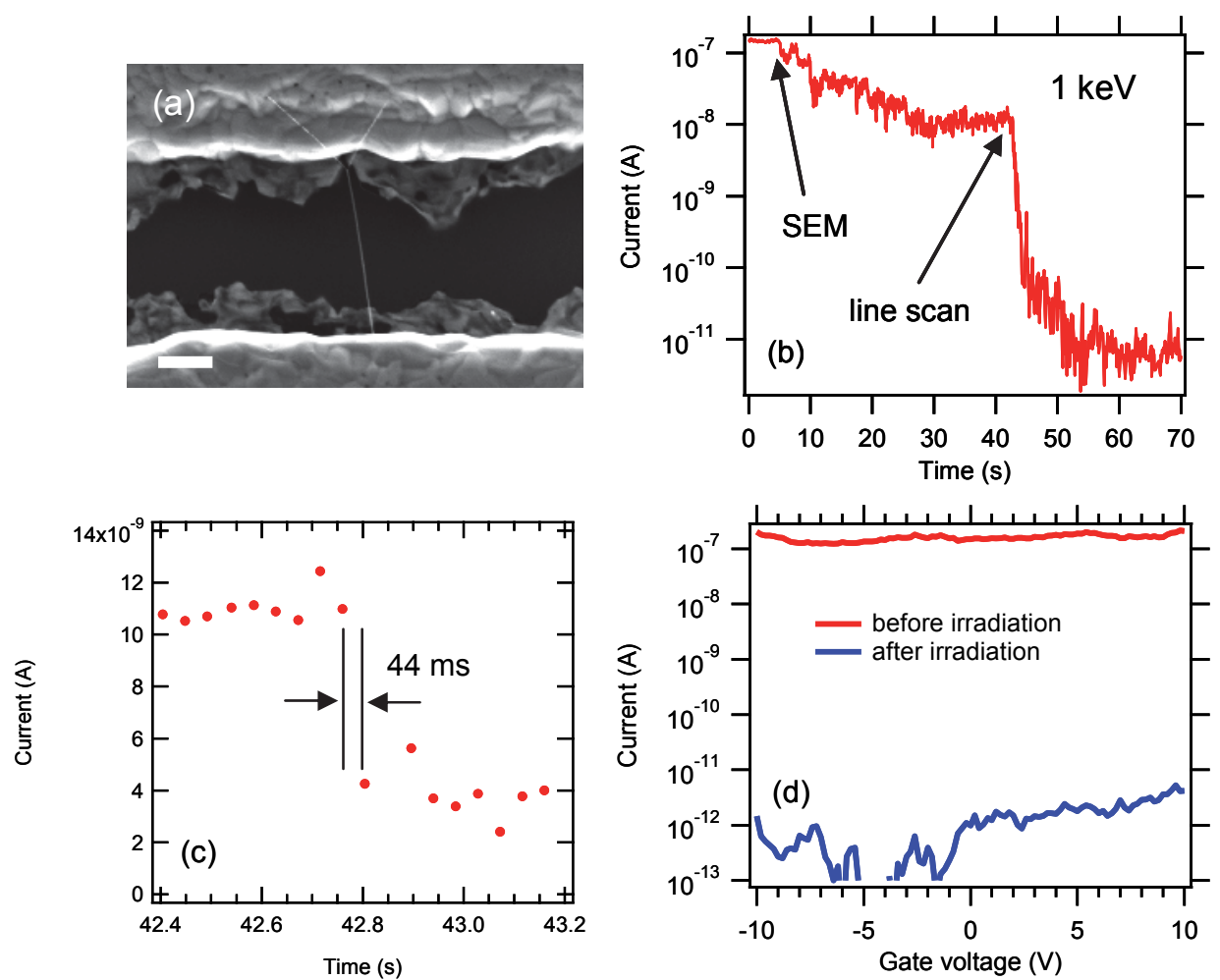

Fig. 4. (a) SEM image of a suspended SWNT device obtained after experiments. The substrate acts as a back-gate electrode. Scale bar: $200 \mathrm{~nm}$. (b) Drain current during SEM observation and line scans. The drain voltage was set to $0.1 \mathrm{~V}$, and the substrate (back-gate) was grounded. The electron energy was $1 \mathrm{keV}$. During the SEM observation, the irradiation dose rate was $1.2 \times 10^{13} \mathrm{~cm}^{-2} \mathrm{~s}^{-1}$ on average in the observation area. The dose rate during the lines scan was $\sim 6 \times 10^{16} \mathrm{~cm}^{-2} \mathrm{~s}^{-1}$. (c) Time evolution of the drain current just before and after the line scan. The data collection was done using the sampling mode of a semiconductor parameter analyzer (Agilent 4156C) and the time interval is not always the same. (d) Gate characteristics of the device before and after the electron irradiation. The drain voltage was $0.1 \mathrm{eV}$.

\section{Characteristics of low-energy irradiation damage}

\subsection{Energy dependence}

The low-energy irradiation damage has been observed in an energy range of several electron-volts to $25 \mathrm{keV}$. One important characteristic of the damage is that, in general, a lower energy is more destructive than a higher energy (Suzuki et al., 2004b), as shown in Fig. 5(a) (except for the energies below $\sim 20 \mathrm{eV}$, where the optical absorption and electron energy loss spectra strongly reflect the specific electronic density of states of graphene). This can be well understood by the fact that the interaction (the cross section of electronic excitation) between a SWCNT and an incident electron (photon) is generally larger at a 

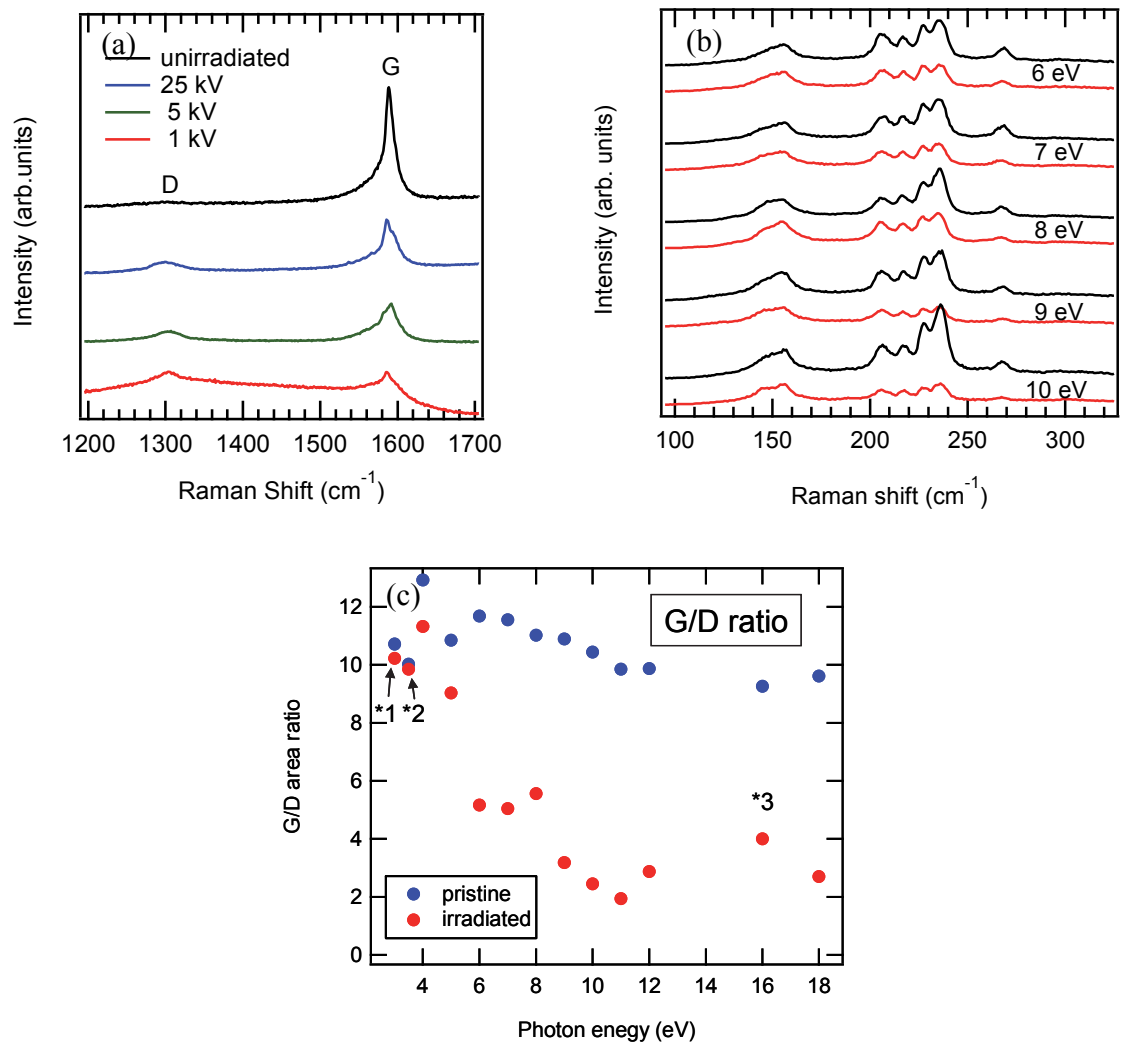

Fig. 5. (a) G and D band spectra of pristine and electron-irradiated SWCNTs, showing the acceleration voltage dependence of electron irradiation damage. The irradiation dose was $2.7 \times 10^{18} \mathrm{~cm}^{-2}$. (b) RBM spectra of pristine SWCNTs (black lines) and SWCNTs illuminated by $6-10 \mathrm{eV}$ photons (red). Because the spectra were originally slightly sample-dependent, spectra before and after the irradiation are shown for each photon energy. (c) G/D area ratio of pristine SWCNTs and SWCNTs irradiated at various photon energies. In (b) and (c), the photon dose was $5 \times 10^{17} \mathrm{~cm}^{-2}$ except for * 2 and ${ }^{*} 3$ in (c). The excitation wavelength was 785 nm. *1) higher order light cut by a pyrex window. ${ }^{*} 2$ ) very intense unmonochromatized light through a pyrex window (hv $\left.\leq 3.5 \mathrm{eV}) .{ }^{*} 3\right)$ The photon dose was $2.5 \times 10^{17} \mathrm{~cm}^{-2}$.

smaller energy. A high-energy electron or photon easily penetrates a SWCNT without any electric excitation. An exception is when the photon energy is tuned to near the $C$ is absorption edge, for which severer damage was observed than the energy below the absorption threshold. However, the resonance effect does not seem to be very prominent, as will be shown in Fig. 11(b).

The existence of the threshold energy is also expected for the low-energy irradiation damage. As seen in the RBM spectra shown in Fig. 5(b), distinct spectral intensity decreases are observed at $\sim 6 \mathrm{eV}$ or larger for semiconducting SWCNTs with diameters of about 1.2-1.0 $\mathrm{nm}$ at $200-240 \mathrm{~cm}^{-1}$ (Suzuki \& Kobayashi, 2008). Decreases in the G/D ratio are also clearly 
observed at $6 \mathrm{eV}$ or higher, as shown in Fig. 5(c). Notably, the damage in metallic SWCNTs with diameters of $\sim 1.6 \mathrm{~nm}$ at $140-160 \mathrm{~cm}^{-1}$ is not clear up to $8 \mathrm{eV}$. This is because of the diameter dependence of low-energy irradiation damage, as discussed in sec. 4.3. In electron (hole) tunneling injection studies using a scanning tunneling microscope (STM), slightly lower threshold energy of $\sim 4 \mathrm{eV}$ has been observed (Yamada et a., 2009). The reason for the discrepancy may be that the threshold energy depends on the diameter, chirality, or detailed defect structures. Anyway the threshold energy of the low-energy irradiation damage seems to be several electron volts.
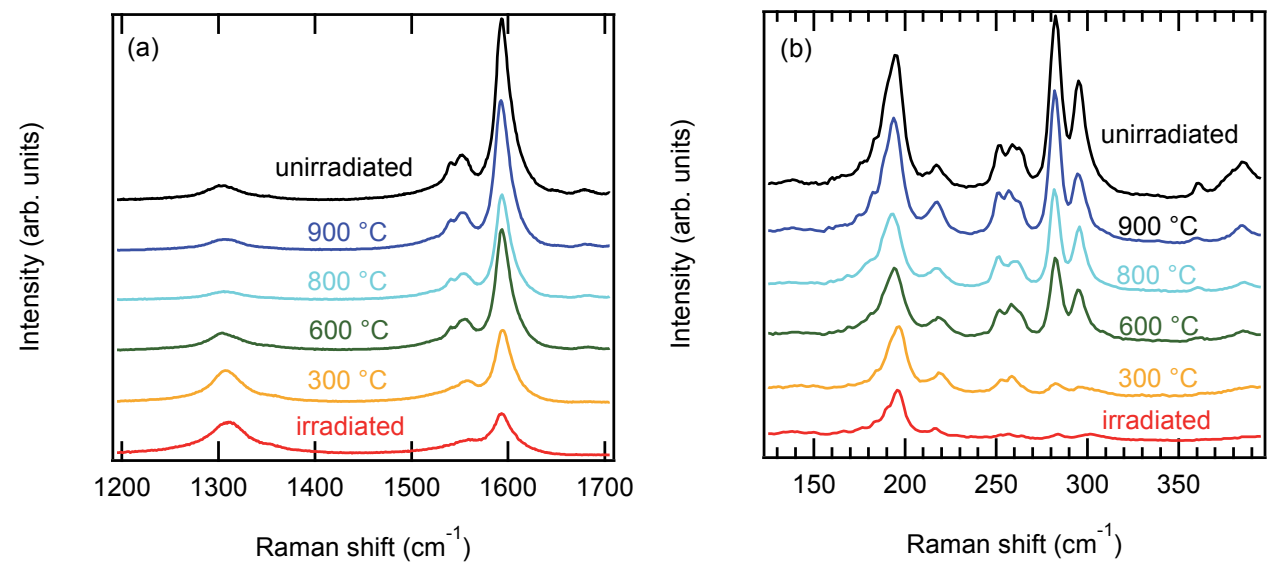

Fig. 6. (a) G and D band, and (b) RBM spectra of unirradiated, photon-irradiated, and annealed SWCNTs. The SWCNTs were irradiated by unmonochromatized synchrotron radiation light $(\mathrm{hv} \leq 1 \mathrm{keV})$ up to a dose of $8 \times 10^{20} \mathrm{~cm}^{-2}$. The excitation wavelength for the Raman measurements was $633 \mathrm{~nm}$.

\subsection{Reversible damage and recovery}

Probably the most important characteristic of the low-energy irradiation damage is the reversibility of the damage and recovery. Figure 6 shows Raman spectra of SWCNTs before and after VUV light irradiation and of SWCNTs annealed at 300, 600, 800, $900{ }^{\circ} \mathrm{C}$. The irradiation caused severe damage and drastically decreased the G/D ratio and the RBM intensities. All the RBM peaks above $200 \mathrm{~cm}^{-1}$ corresponding to a diameter less than $\sim 1.2 \mathrm{~nm}$ almost completely disappeared. However, the annealing at 300,600 , and $800{ }^{\circ} \mathrm{C}$ gradually recovered the spectra, and at $900^{\circ} \mathrm{C}$, all the peaks including the once disappeared peaks are almost fully recovered. I would like to point out once again that the spectra were not normalized at all. Thus, the results reveal that not only the spectral shape but also the spectral intensity itself is almost fully recovers by annealing.

The reversible damage and recovery is also observed in the electric properties. As mentioned in sec. 3.3, the low-energy irradiation damage almost extinguishes the electric conductivity. However, the extinguished conductivity is also fully recoverd by annealing, as shown in Fig. 7(a). An originally metallic SWCNT device was intensively irradiated in a SEM using the line scan mode. The irradiation extinguished the conductivity and made the SWCNT almost insulating. However, the conductivity was fully recovered by annealing in a 
vacuum at $300{ }^{\circ} \mathrm{C}$. Moreover, the reversibility can be observed repeatedly, as shown in Fig. 7(b). The complete reversibility of the electric properties has also been observed by another group, although they attribute the conductivity decrease and recovery to substrate charging and its release (Marquardt et al., 2008. and Vijayaraghavan et al., 2010). I will discuss this issue later in sec. 7.4.
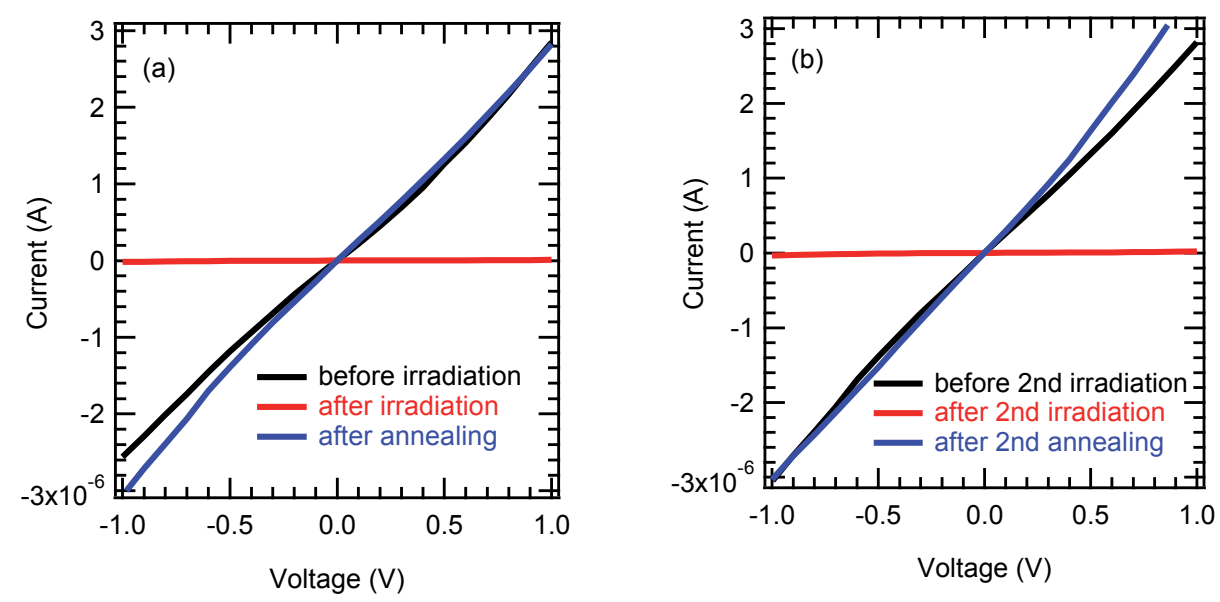

Fig. 7. (a) Electric properties of a SWCNT device before and after the first electron irradiation and after annealing. The SWCNT was intensively irradiated by electrons of 20 $\mathrm{keV}$ up to a dose of $1 \times 10^{20} \mathrm{~cm}^{-2}$. Then, the irradiated device was annealed at $300{ }^{\circ} \mathrm{C}$ in $\mathrm{Ar}$ atmosphere for $30 \mathrm{~min}$. (b) The electric properties of the same SWCNT device before and after the second irradiation and after the second annealing. The SWCNT was intensively irradiated again by $20-\mathrm{keV}$ electrons up to a dose of $1.7 \times 10^{20} \mathrm{~cm}^{-2}$. After the irradiation, the SWCNT was annealed at $300^{\circ} \mathrm{C}$ for $30 \mathrm{~min}$ and fully recovered again.

The reversibility of the damage and recovery indicates that the damage is not accompanied by a reduction of carbon atoms and that the number of carbon atoms is preserved. Recently, Mera et al. directly measured ion desorption from SWCNTs under soft X-ray illumination (Mera et al, 2010). They also excluded emission of carbon atoms from the SWCNTs.

\subsection{Diameter dependence}

Another important characteristic of the low-energy irradiation damage is that strong diameter dependence is observed when the irradiation is done at room temperature or above (Suzuki and Kobayashi, 2006). For example, in the RBM and PL spectra shown in Figs. 1, 2, 5, and 6, we can clearly see the diameter dependence of the damage; that is, thinner SWCNTs are more severely damaged. Especially, in Fig. 1(c), we can see a large difference in the extent of the damage due to very small diameter difference. The SWNTs observed at about 1151 and $1172 \mathrm{~nm}$ can be assigned to $(12,1)$ and $(11,3)$ tubes having diameters of 0.995 and $1.014 \mathrm{~nm}$, respectively. These two peaks were considerably weakened by the photon irradiation at $250{ }^{\circ} \mathrm{C}$. On the other hand, the occurrence of the damage was not obvious for thicker SWNTs after the same irradiation dose at $250{ }^{\circ} \mathrm{C}$. The peaks at $1224 \mathrm{~nm}$ is assigned to $(10,5)$ tubes having a diameter of $1.050 \mathrm{~nm}$. The diameter 
difference between the considerably damaged $(11,3)$ and hardly damaged $(10,5)$ tubes is only $0.037 \mathrm{~nm}$. It is very interesting that such a small diameter difference results in the distinctly different sensitivity to the irradiation.
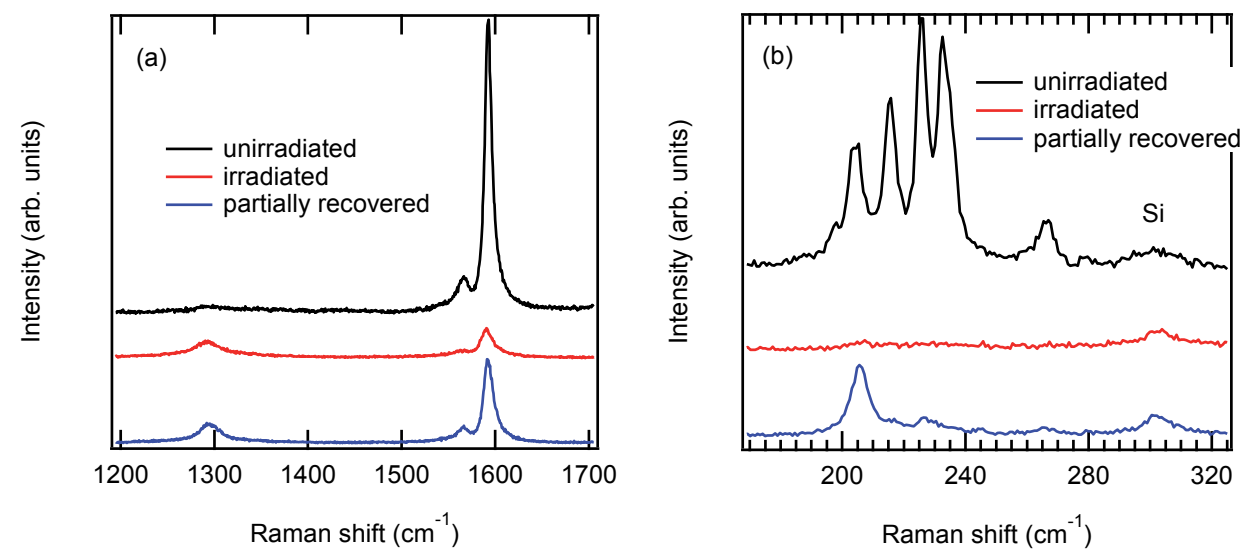

Fig. 8. (a) G and D band and (b) RBM spectra of pristine, irradiated, and partially recovered SWCNTs. The SWCNTs were irradiated by 1-keV electrons up to a dose of $8 \times 10^{17} \mathrm{~cm}^{-2}$. Then, the damaged SWCNTs were partially recovered by annealing at $400{ }^{\circ} \mathrm{C}$ in $\mathrm{Ar}$ atmosphere for $30 \mathrm{~min}$. The excitation wavelength was $785 \mathrm{~nm}$. In (b), the hump at $\sim 300 \mathrm{~cm}^{-1}$ is from the $\mathrm{Si}$ substrate.

The diameter dependence is observed in the recovery process, as shown in Fig. 8. The electron irradiation largely decreased the G/D ratio (a) and once almost completely extinguished all of the RBM peaks (b). The sample was partially recovered by annealing at $400{ }^{\circ} \mathrm{C}$. Then, only the peak at about $205 \mathrm{~cm}^{-1}$, corresponding to the thickest SWNTs among the initially observed ones, significantly recovered.

The diameter dependence of damage is more or less also observed in knock-on damage (Krasheninnikov \& Nordlund, 2010) and damage by radicals (Yang et al., 2006, Zhang et al., $2006 \mathrm{~b})$. However, the diameter dependence of low-energy irradiation damage is more prominent, as mentioned above. The damage caused by knock-on collision and radicals also occurs in thick MWCNTs and graphite, but the low-energy irradiation damage has not. Also noteworthy is that the diameter dependence is not prominent when SWCNTs are irradiated at low temperature, as shown in the next section.

\subsection{Temperature dependence of the damage}

Severer damage is observed at lower temperatures (Suzuki \& Kobayashi, 2007a). As shown in Fig. 1, the irradiation at $250{ }^{\circ} \mathrm{C}$ results in much less damage than at $22{ }^{\circ} \mathrm{C}$. The temperature dependence is seen at lower temperatures, and less damage is observed at -27 ${ }^{\circ} \mathrm{C}$ than at $-267{ }^{\circ} \mathrm{C}(6 \mathrm{~K})$, as shown in Fig. 9. These results suggest that low-energy irradiation-induced defects can be healed even at $-27^{\circ} \mathrm{C}$. In fact, the electric conductivity of irradiated SWCNTs gradually recovers at room temperature, as shown in Fig. 10 (Suzuki \& Kobayashi, 2007). The temperature dependence of the damage is completely opposite to that observed in gas phase reactions (An et al., 2002, Zhang et al., 2006a). 

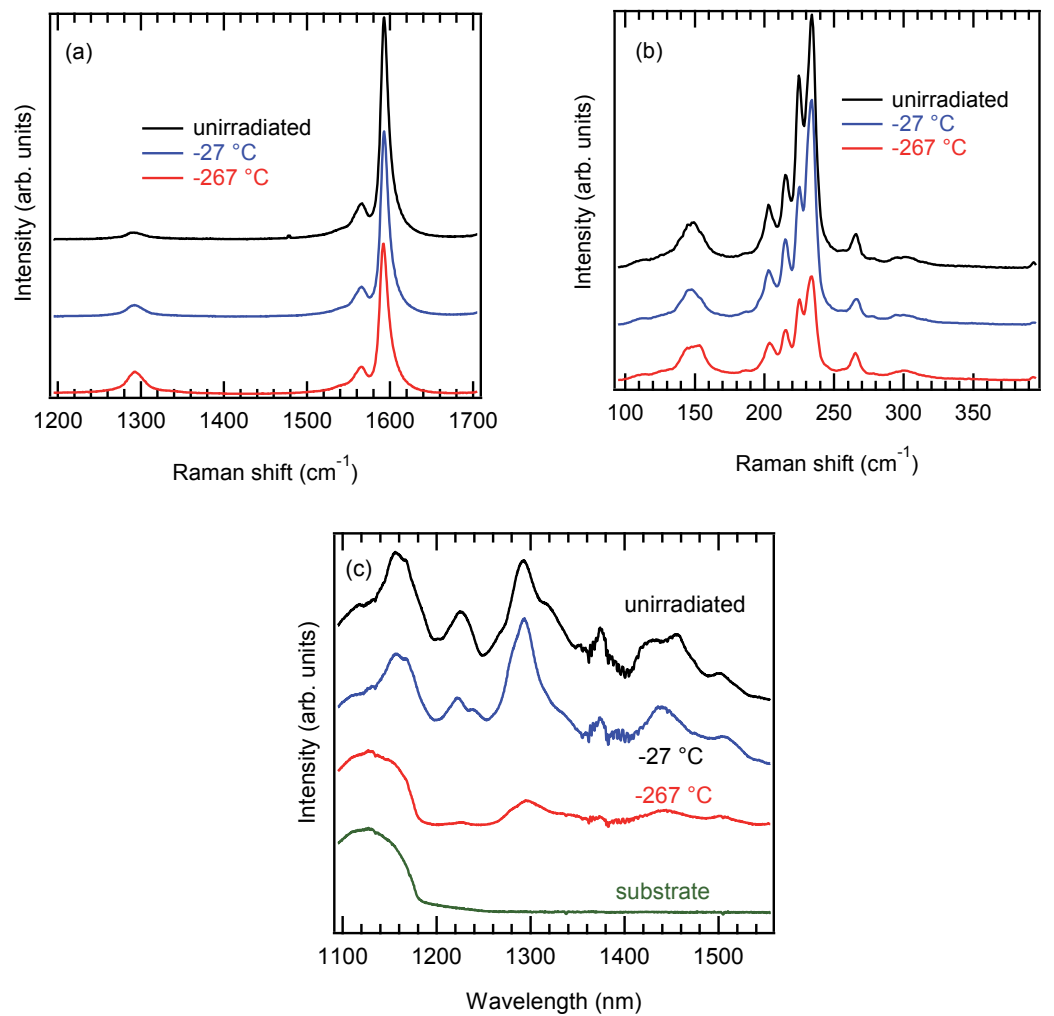

Fig. 9. (a) G and D band, (b) RBM, and (c) PL spectra of pristine SWCNTs and of SWCNTs irradiated at -27 , and $-267^{\circ} \mathrm{C}$. The SWCNTs were irradiated by $40-\mathrm{eV}$ photons up to a dose of $7.2 \times 10^{17} \mathrm{~cm}^{-2}$. The excitation wavelength was $785 \mathrm{~nm}$. In (b), the hump at $\sim 300 \mathrm{~cm}^{-1}$ is from the Si substrate.

Fig. 9(b) also shows that the diameter dependence of the damage is less prominent at $-27^{\circ} \mathrm{C}$, meaning that the recovery of all the observed SWCNTs is almost completely forbidden at this temperature, regardless of the diameter. In other words, the less damage in a thicker SWCNT observed at room temperature and above is a consequence of the fact that a defect created in the thicker SWCNT can be more quickly healed by the thermal energy at the irradiation temperature. The diameter dependence can mainly be ascribed to the diameter dependence of the defect healing rather than to that of defect creation.

\subsection{Activation energy of defect healing}

The recovery of the damage at room temperature or below suggests that the activation energy of the defect healing is quite small. We have proposed a simple method for determining the activation energy of defect healing in SWCNTs (Suzuki et al., 2010). For example, recovery curves of the G/D ratio can be used for the analysis. In Fig. 11(a), we show Raman spectra of SWCNTs before and after irradiation and after 2, 14, and $64 \mathrm{~min}$ annealing at $240{ }^{\circ} \mathrm{C}$. The annealing gradually recovered the irradiated SWCNTs. From these 
measurements, we obtained recovery curves of the G/D ratio at several temperatures, as shown in Fig. 11(b). The reason for the relatively small values of the G/D ratio is that we adopted the area ratio rather than the peak height ratio, in order to decrease static errors.
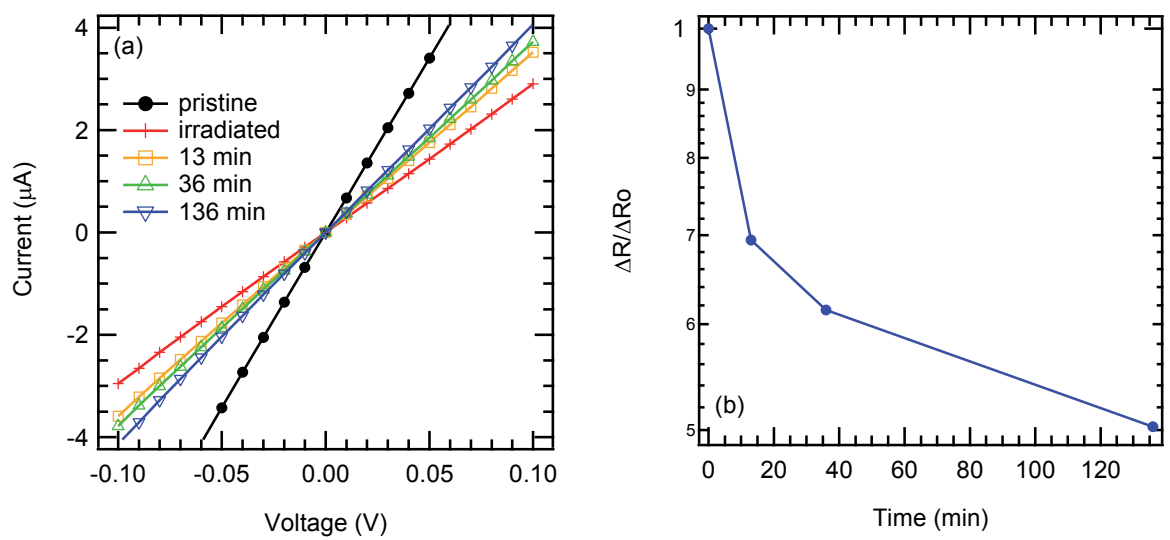

Fig. 10. (a) Current-voltage characteristics of a SWCNT device before and just after electron irradiation and 13, 36, $136 \mathrm{~min}$ after the irradiation. The electron energy and irradiation dose were $20 \mathrm{keV}$ and $1.8 \times 10^{15} \mathrm{~cm}^{-2}$,respectively. (b) Time evolution of the irradiation-induced resistance $(\Delta \mathrm{R})$ at $22{ }^{\circ} \mathrm{C}$. The initial value of the irradiation-induced resistance $(\Delta \mathrm{Ro})$ was 19.8 $\mathrm{k} \Omega$.

The activation energy $E_{\mathrm{a}}$ of defect healing is given by

$$
E_{a}=\frac{k}{\left(T_{1}^{-1}-T_{2}^{-1}\right)} \ln \frac{t_{1}}{t_{2}}
$$

where $t_{1}$ and $t_{2}$ are annealing times during which the G/D ratio $R$ increases from $R_{1}$ to $R_{2}$. Here, it is sufficient that the G/D ratio is just a monotonic function of the defect density (We do not have to assume a specific relation between the G/D ratio and defect density, such as that the G/D ratio is inversely proportional to the defect density). The activation energy seems to depend on the extent of the damage. In the region of $2.8 \leq R \leq 3.0$ (heavily damaged), we obtained an activation energy value of $1.4 \pm 0.2 \mathrm{eV}$, as shown in Fig. 11(c), from the recovery curves at 140 and $120^{\circ} \mathrm{C}$ and eq. (1), whereas at $6 \leq R \leq 6.5$ (lightly damaged), a smaller value of $0.7 \pm 0.2 \mathrm{eV}$ was obtained. I would like to mention that these values may be affected by gas absorption at defect sites, because the SWCNTs were once exposed to air after the irradiation. Anyway, the values are small enough for the defects to be healed at moderate temperatures.

Interestingly, although a partial recovery of low-energy irradiation damage at room temperature is easily observed in the electric properties (Fig. 10), it has not been observed in Raman spectra. Once we obtain the activation energy value, we can estimate the recovery curve at a given temperature $T_{3}$. Using the recovery curve $R_{1}\left(R_{2}\right)$ at $T_{1}\left(T_{2}\right)$, the recovery curve at $T_{3}$ is given by

$$
R_{3}\left(t_{3}\right)=R_{1,2}\left(t_{3} \exp \left[\frac{E_{a}}{k}\left(T_{1,2}^{-1}-T_{3}^{-1}\right)\right]\right) .
$$



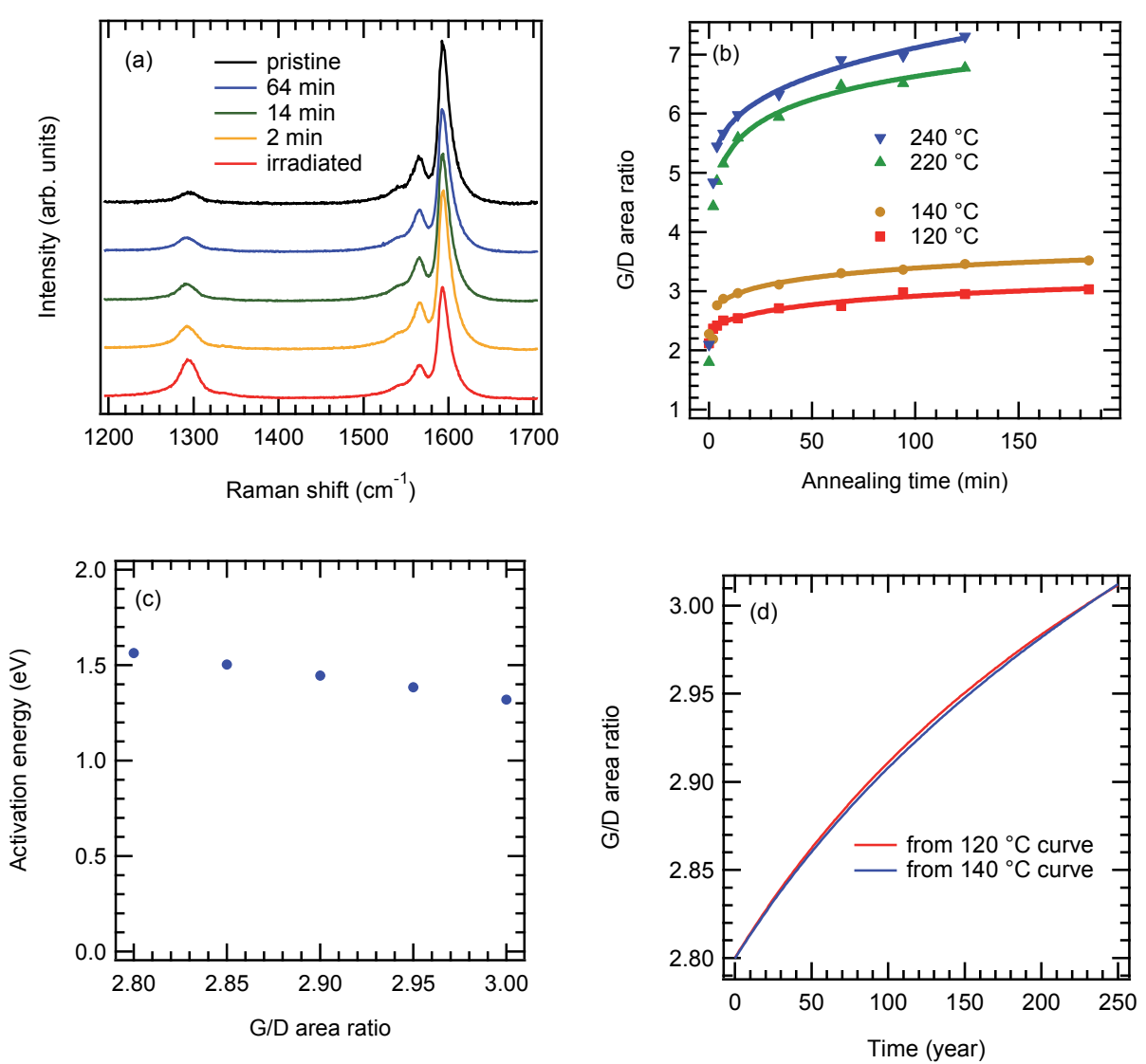

Fig. 11. (a) Raman spectra of SWCNTs before and after electron irradiation and after annealing at $240{ }^{\circ} \mathrm{C}$. The electron energy and dose were $20 \mathrm{keV}$, and $8 \times 10^{16} \mathrm{~cm}^{-2}$, respectively. The excitation wavelength was $785 \mathrm{~nm}$. (b) Recovery curves of the G/D area ratio otbained at several annealing temperatures. (c) Activation energy of the defect healing obtained from the recovery curves at 140 and $120^{\circ} \mathrm{C}$. (d) Simulated recovery curves of the $\mathrm{G} / \mathrm{D}$ ratio at room temperature $\left(20^{\circ} \mathrm{C}\right)$ obtained from the recovery curves at 120 and $140{ }^{\circ} \mathrm{C}$ in (b), respectively.

Fig. $11(\mathrm{~d})$ shows the recovery curves at $20^{\circ} \mathrm{C}$ simulated from the experimental recovery curves at 140 and $120^{\circ} \mathrm{C}$ and eq. (2). The two independently obtained curves are almost consistent. Note that the unit of the horizontal axes is "year". Recovery of the G/D ratio from 2.8 to 3.0 at $20^{\circ} \mathrm{C}$ would take about 230 years. Similarly, the recovery from 6.0 to 6.5 at $20{ }^{\circ} \mathrm{C}$ was estimated to take about 7 years (Suzuki et al., 2010). Thus, the recovery would be much too slow to observe at room temperature in usual experiments. The very long recovery time at room temperature is a consequence of the relatively slow recovery at elevated temperatures in Raman spectra. On the other hand, the recovery of the electric properties is much more rapid. Annealing at $300{ }^{\circ} \mathrm{C}$ for $30 \mathrm{~min}$ often results in recovery of conductivity of several orders of magnitude, as already shown in Fig. 7. 

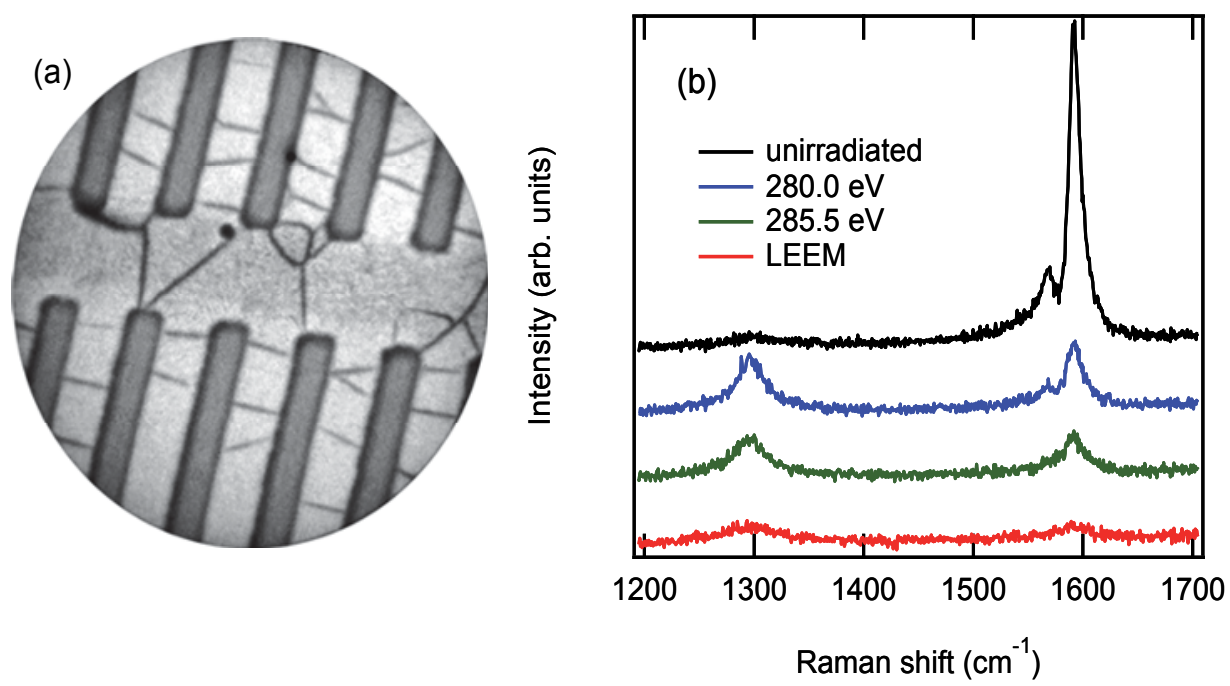

Fig. 12. (a) LEEM image of suspended SWNTs on a patterned Si substrate. (b) G and D band Raman spectra of unirradiated and soft X-ray-illuminated (280. 0 and $285.5 \mathrm{eV})$ SWCNTs and electron-irradiated $(\sim 20 \mathrm{eV})$ SWCNTs. The excitation wavelength was $785 \mathrm{~nm}$. The soft X-ray illumination was done at BL-27SU at SPring-8, Hyogo, Japan. The electron irradiation was a consequence of LEEM observation. The soft $X$-ray and electron irradiations were done after thorough degassing.

Though we evaluate the activation energy from the G/D ratio here, any other quantity that is a monotonic function of the defect density can basically be used for the analyses. This method can also be used to analyze other kinds of defects and desorption barriers of chemisorbed atoms or molecules on SWCNTs.

\subsection{Occurrence of the damage in an ultra-high vacuum}

As I mentioned in sec. 2, the low-energy irradiation damage is caused by the irradiation itself. We found that the damage does not depend on the remnant gas pressure at $\sim 10^{-4} \mathrm{~Pa}$ or below (Suzuki et al., 2008). The low-energy irradiation damage has been observed in a surface-science-grade ultra-high vacuum (UHV) of $\sim 1 \times 10^{-8}$ Pa by VUV light illumination (Suzuki \& Kobayashi, 2006a. Suzuki \& Kobayashi, 2007a, Mera et al., 2009. Mera et al., 2010.), electron beam irradiation (Arima et al., 2009.), and electron (hole) injection from a STM tip (Berthe et al., 2007. Yamada et al., 2009). The damage occurs as ever when SWCNTs are thoroughly degassed in a UHV before irradiation. Other examples of occurrence of the damages in UHV surface analysis systems are shown in Fig. 12(b). The SWCNTs were irradiated by electrons during LEEM observation [Fig. 12(a)] or by soft X-rays at a photoemission spectroscopy beamline attached to a synchrotron radiation ring. The damage to SWCNTs is especially severe in LEEM observation using very low-energy electrons of several tens electron volts, due to the energy dependence of the damage (sec. 4.1). In an UHV, no irradiation-induced change is observed even in high-energy-resolution 
photoemission spectroscopy (Suzuki et al., 2004a), indicating that chemical reactions with gas molecules are negligible. Nevertheless, very severe damage is observed in Raman spectra.

\subsection{Structure dependence}

A low-energy electron and photon can easily dissociate a small molecule (for example, photodissociation). On the other hand, such low-energy irradiation damage (or structural change) is not commonly observed inside the bulk of a metal or semiconductor. Actually, it has not been reported for graphite. Very interestingly, even among CNTs, the damage has been reported for SWCNTs but not for MWCNTs. An electron irradiation experiment in an SEM has shown that the irradiation causes no reduction of the conductivity of MWCNTs with a diameter of $\sim 10 \mathrm{~nm}$ (Bachtold et al., 1998. Hobara et al., 2004). The irradiation conditions used in those studies $\left(4 \mathrm{C} \cdot \mathrm{cm}^{-2}\right.$ of $20-\mathrm{keV}$ and $20 \mathrm{C} \cdot \mathrm{cm}^{-2}$ of $10-\mathrm{keV}$ electrons) roughly correspond to 10 to 1000 fold of a value that can cause a SWCNT conductivity decrease of a few orders of magnitude (Suzuki, 2011). Thus, the damage seems to be specific to SWCNTs or thin CNTs with a diameter of $\sim 1 \mathrm{~nm}$. Even among SWCNTs, the extent of the damage strongly depends on the diameter: Thinner SWCNTs are more severely damaged, as discussed in section 4.3.

The diameter dependence of the damage may explain the occurrence of the damage in SWCNTs and its absence in MWCNTs and graphite. Considering that the damage strongly depends on the diameter among SWCNTs, it would be possible that a MWCNT of 10-nm diameter is no longer damaged by low-energy irradiation at room temperature. If the occurrence and the absence of the damage originate in the diameter difference, we can expect that strain in the sidewall plays an essential role in the defect formation or its stabilization. Alternatively, it is interesting to view the occurrence and absence of the damage in terms of dimensionality. Graphite, in which the damage does not occur, is a three-dimensional material, and a SWCNT, in which the damage occurs, is a onedimensional material. Notably, it has been well established that structural changes occur in zero-dimensional fullerenes by photon and electron irradiation (Zhao et al., 1994. Onoe et al., 2003). This is generally described as "polymerization" instead of damage, because the irradiation causes chemical bonds to form between neighboring fullerenes. The structural change can be reversibly restored by annealing, exactly like the low-energy irradiation damage of a SWCNT. The electronic states, which spread in the whole crystal in a bulk material, should be localized in low-dimensional materials or nanomaterials, and the degrees of freedom of atomic movement should become larger. Thus, in low-dimensional materials or nanomaterials, local structural change would easily occur with low-energy irradiation and the defect structure would be stabilized (See also sec. 6).

In terms of the relation between the damage and structure, it is very interesting to explore whether the damage occurs in graphene, which is a two-dimensional material and can be considered to be a SWCNT of infinite diameter. Zhou et al. reported that soft $x$-ray illumination damages graphene, on the basis of their C 1s x-ray absorption and Raman spectroscopy results (Zhou et al., 2009). Very interestingly, the illumination effects increased with a decreasing number of layers of exfoliated graphene and were negligible even for monolayer epitaxial graphene on $\mathrm{SiC}$, which has a relatively strong interaction with the substrate. These results suggest that low dimensionality is strongly related to the lowenergy irradiation damage. 

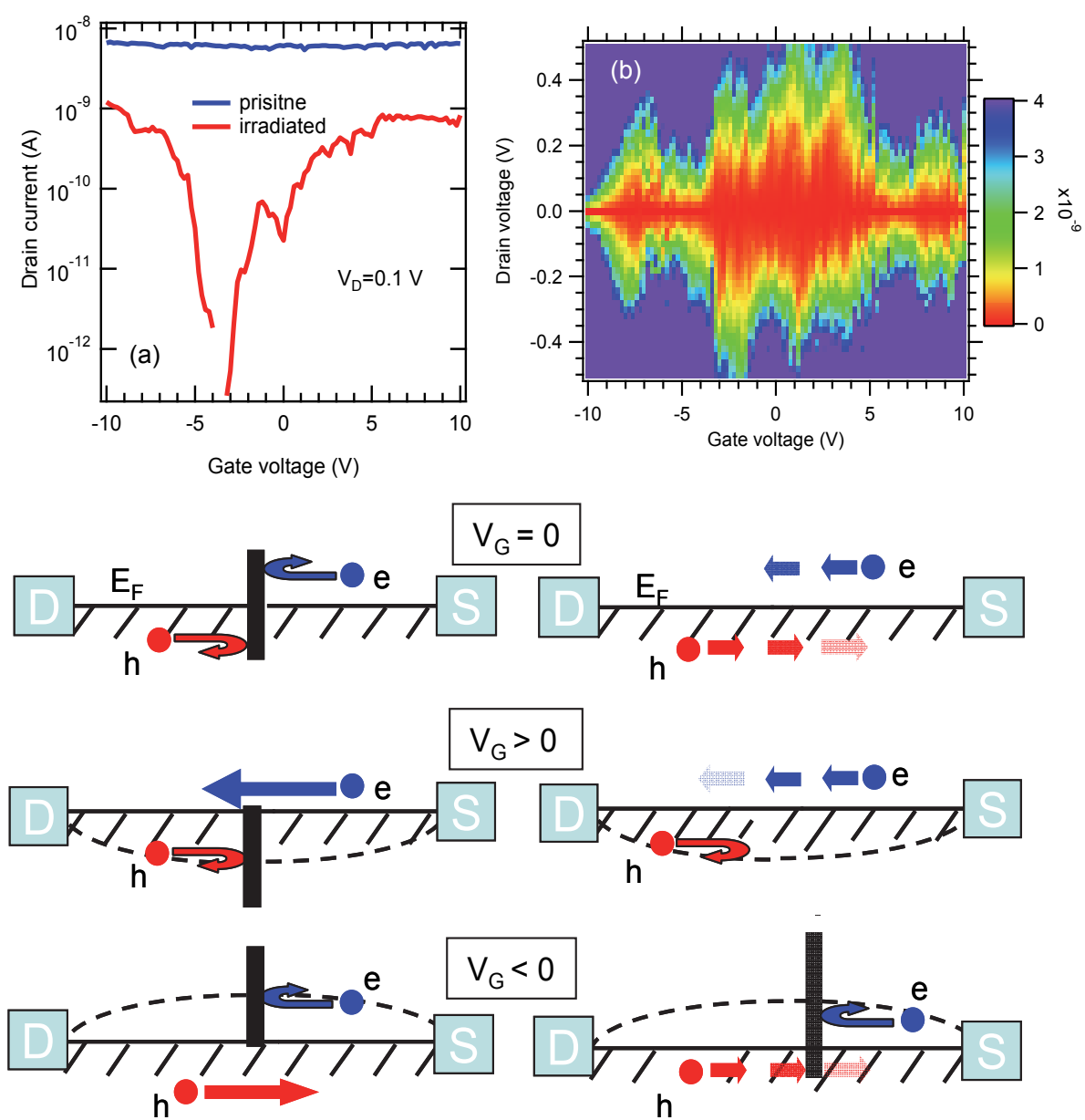

(c)

(d)

Fig. 13. (a) Room-temperature gate voltage characteristics of a SWCNT device before and after electron irardiation. The SWCNT was once scanned by an electron beam of $100 \mathrm{pA}$. The electron energy and scan speed were $20 \mathrm{keV}$ and $400 \mathrm{~nm} / \mathrm{s}$, respectively. (b) Roomtemperature Coulomb diamond characteristics of a SWCNT device before and after electron irradiation. The SWCNT was once scanned by an electron beam of $500 \mathrm{pA}$. The electron energy and scan speed were $20 \mathrm{keV}$ and $400 \mathrm{~nm} / \mathrm{s}$, respectively. (c) Schematic explanations of the defect-induced semiconductng properites. (d) Schematic explanations of the defectinduced Coulomb oscillation properites.

\section{Metal-semiconductor transition of a SWCNT-FET induced by defects}

As mentioned in sec. 3.3, intensive irradiation finally makes a SWCNT almost insulating. However, when the damage is moderate, a metal-semiconductor transition of the electric properties is often observed. In our early study, we irradiated the whole device in a SEM and 
observed the conversion of the electric properties at $28 \mathrm{~K}$ (Vijayaraghavan et al., 2005). Further irradiation caused an increase of the nominal band gap observed in the low-temperature electric properties. More recently, we succeeded in converting the room-temperature device characteristics from metallic to semiconducting by local irradiation using an electron beam lithography system (Suzuki et al., 2008). Before the irradiation, the device characteristics were almost gate-independent, which is a common feature of a metallic SWCNT. A part of a metallic SWCNT was once scanned by an electron beam. Then, the room-temperature gate characteristics of the device were converted to semiconducting, as shown in Fig. 12(a). After the irradiation, ambipolar semiconducting gate characteristics were clearly observed.

Room-temperature Coulomb oscillations have been observed when defects form a small dot in a SWCNT channel (Matsumoto et al., 2003). The low-energy irradiation damage can also be used to fabricate such small dots intentionally. As shown in Fig. 12(b), after irradiation, multi-dot Coulomb oscillation properties are sometimes observed at room temperature.

A schematic model of a possible mechanism for the irradiation-induced semiconducting properties is shown in Fig. 12(c) (Kanzaki et al., 2007. Suzuki et al., 2008). The temperature dependence of device characteristics after irradiation shows that an energy barrier for carriers is formed in the SWCNT channel. The barrier height observed in the electric properties reaches about $0.6 \mathrm{eV}$, when irradiation-induced semiconducting properties are observed at room temperature. Recently, a STM study more directly showed that a local band gap is actually formed in a metallic SWCNT by a carrier injection-induced defect (Yamada et al., 2009). This suggests that the defect-induced local band gap opening is the origin of the barrier. The carrier transport is inhibited by the barrier at the gate voltage of around $0 \mathrm{~V}$. The device still turns on at large gate voltage. This can be reasonably explained in terms of gate-induced band bending in a metallic SWCNT. The density of states near the Fermi level of a metallic SWCNT is very small. Owing to the small density of states near the Fermi level, we can bend the band by applying gate voltage and reduce the effective barrier height for an electron. At sufficiently large gate voltage, the device will turn on. Thus, the metal-semiconductor transition is explained by the defect-induced barrier formation and gate-induced band bending. When Coulomb oscillation is observed, the defects seem to act as tunneling barriers, as schematically shown in Fig. 12(d). In this case, tiny multi-dots divided by the defects seem to be formed in the vicinity of the irradiated part.

The defect-induced conversion of the electric properties seems to be caused by defects formed by other methods. In fact, conversion of the electric properties from metallic to semiconducting also occurs when defects are induced by plasma treatment of metallic SWCNT-FETs. More interestingly, the defect-induced semiconducting electric properties well explain the fact that the ratio of "semiconducting" SWCNTs that act as FETs has been reported to strongly depend on the growth method (Suzuki et al., 2008, Mizutani et al., 2009). The plasma-enhanced CVD method has been reported to produce preferentially semiconducting SWCNTs, and the ratio of semiconducting SWCNTs has been reported to reach about 90 \% (Li et al., 2004. Ohnaka et al., 2006) or even 97 \% (Mizutani et al., 2009). On the other hand, for the laser ablation method, which generally produces high-quality SWCNTs, the semiconducting SWCNT ratio was evaluated to be quite small, about $30 \%$ (Li et al., 2004). I think that the growth method dependence of the "semiconducting" SWCNT ratio is mainly due to the growth method dependence of defect density. Distinguishing whether the electronic structure is metallic or semiconducting by electric measurements may be inconclusive, especially when the SWCNT shows "semiconducting" properties. 


\section{Mechanism of the low-energy irradiation damage}

The low-energy irradiation damage can be caused by $10-\mathrm{eV}$ photons, which have very small momentum. This indicates that the momentum of an incident particle would have no essential role in the damage, which is in remarkable contrast to knock-on damage. Thus, the defect formation would be due to bond breaking, which follows an electronic excitation by the energy of the incident particle. An energy of $\sim 10 \mathrm{eV}$ is still high enough to cut C-C bonds. Thus, it is reasonable that low-energy irradiation creates a defect with finite probability if the defect structure is stable and the lifetime is long enough. A simple example of this kind of structural change is photodissociation of a molecule. A bond breaking following electronic excitation can easily dissociate a small molecule In a bulk crystal, on the other hand, even the breaking of several bonds would result in immediate re-bonding without any structural change because an atom has very little freedom of displacement due to the existence of surrounding atoms. The situation in a SWCNT is one between a molecule and bulk. More than one bond breaking would be necessary to stabilize the defect. Among related carbon materials, low-energy electron and photon irradiation-induced structural change (polymerization) is known to occur for fullerenes. On the other hand, the damage has not been reported for graphite or MWCNTs, as discussed in sec. 4.7.

Interestingly, Yamada et al. (Yamada et al., 2009) have proposed, on the basis of thier STM results, that carrier injection first creates primary defects whose lifetime is very short $(<50$ $\mathrm{ms}$ ). Most of them are quickly annihilated and the structure is restored. However, in rare cases, a primary defect fails to recover and a stable defect is created. The quantum efficiency of the primary defect formation was evaluated to be $2 \times 10^{-10}$ at a bias voltage of $3.5 \mathrm{~V}$ near the defect creation threshold.

The detailed atomic structure of a low-energy irradiation-induced defect is not clear at present. A detectable change has not been observed even with microscopy techniques, such as SEM. This is one of the main reasons that the low-energy irradiation had not been recognized for such a long time, although SEM had been commonly used for characterizing SWCNTs since their discovery. Our previous TEM observation showed that the tube wall is not clearly destroyed regardless of severe damage (Suzuki et al., 2005b). The Stone-Wales defect, which is formed by a C-C bond rotation, is consistent with the conservation of the number of carbon atoms. However, the stable structure seems to contradict the relatively small activation energy and healing at a moderate temperature or even at room temperature or below. Another possible defect is a vacancy in the tube wall with a migratory $\mathrm{C}$ adatom on the surface. The observed activation energies $(0.7-1.4 \mathrm{eV}$, sec. 4.5$)$ are very close to the $\mathrm{C}$ adatom migration energies, which are theoretically predicted to depend on the SWCNT diameter and to be 0.6 to $1.3 \mathrm{eV}$ (Krasheninnikov et al., 2004). However, a high-resolution TEM observation has shown that annihilation of the vacancy and migratory adatom is governed by the recombination barrier rather than by the adatom migration barrier itself (Hashimoto et al., 2004). The existence of such a vacancy-adatom defect (with the adatom bounded in the vicinity of the vacancy) was also strongly suggested by a scanning tunneling microscopy study (Lee et al., 2005). The vacancy-adatom defect is simply formed by breaking two bonds of a $\mathrm{C}$ atom. The recombination barrier of the vacancy-adatom defect in SWCNTs has been calculated to be $\sim 1-2 \mathrm{eV}$ (Okada, 2007), which is rather close to the observed activation energies. Determining the presice defect structure is a future issue. 


\section{Other types of damage and irradiation-induced phenomena}

The low-energy irradiation damage is often confused with other types of damage and irradiation-induced phenomena. Here, I would like to summarize differences in the defect characteristics of low-energy irradiation damage and other damages.

\subsection{Knock-on damage}

Knock-on damage is caused by ballistic ejection of an atom from a solid by an incident particle. Thus, the damage is accompanied by a loss of SWCNT mass. The displacement energy (kinetic energy at which an atom can escape from the solid.) in a SWCNT is considered to depend on the diameter and to be $15-20 \mathrm{eV}$. However, when the incident particle is an electron whose mass is much smaller than a carbon atom, the threshold energy becomes $\sim 80 \mathrm{keV}$ from the energy and momentum conservation laws. The threshold energy for a photon will be much larger. Thus, the energy at which knock-on damage is observed is much larger than that at which the low-energy irradiation damage is normally observed. Knock-on damage occurs in MWCNTs and graphite. For a recent review focusing on carbon and other nanomaterials, see (Krasheninnikov, 2010).

\subsection{Contaminant effects}

Low-energy irradiation often causes hydrocarbon contaminants to adhere to the sample surface. The contaminant adhesion is prominent in a conventional SEM, in which a UHV is usually unavailable. At the electron energy where severe damage is observed $(1 \mathrm{keV}$ or smaller), severe contaminant adhesion is also observed. This is because the interaction both between electrons and a SWCNT and between electron and hydrocarbon gases are strong at such a low energy. However, effects caused by the damage and by the contaminant adhesion can be easily distinguished by annealing. The contaminants do not sublimate even at $\sim 900{ }^{\circ} \mathrm{C}$, whereas the low-energy irradiation damage can be recovered by annealing at moderate temperatures, as discussed in sec. 4 . In our experiments, it is rather difficult to detect contaminant effects because the spectra or electric properties of pristine SWCNTs and SWCNTs recovered from the damage are almost identical (see Figs. 6 and 7), although I do not deny that the contaminants cause some so-called environmental effects (Ohno, 2010).

\subsection{Damage by radicals}

Irradiation in remnant gases can also cause radical-induced etching, which is the opposite of the contaminant adhesion. For example, the cutting of MWCNTs has been clearly demonstrated under gas atmosphere formed by intentional gas bleeding (Yuzvinsky et al., 2005). In this way, the damage by radicals is generally accompanied by etching, which eventually cuts and eliminates CNTs. Thus, this damage can not be fully recovered. In some literatures, reversible chemisorption and desorption on SWCNT or graphene has been suggested. However, spectra before chemisorption and after desorption by annealing are often compared after arbitrary normalization. This damage also occurs in thick MWCNTs and graphite, although some diameter dependence of the damage is observed (Yang et al., 2006, Zhang et al., 2006b). Metallic SWCNTs are preferentially damaged by radicals (Yang et al., 2006. Zhang et al., 2006b), although such preference has not been observed for lowenergy irradiation damage. Moreover, severer damage is observed at higher temperatures due to more activated chemical reactions (An et al., 2002. Zhang, 2006a). This is entirely opposite to the low-energy irradiation damage (sec. 4.4). Of course, the radical effect 
becomes severer at higher pressures and negligible in a UHV. Reader should recall that lowenergy irradiation damage occurs in a UHV after thorough degassing, although no indication of chemical reaction is observed (sec. 4.6).

In a standard SEM in which the adhesion of contaminants occurs, the radical effects seem to be less important. Otherwise, the contaminants would not adhere due to etching. According to my experience, intensive irradiation in a conventional SEM can not cut or eliminate even a SWCNT (Fig. 7). I suppose that the radical effects are largely suppressed due to the adhesion of contaminants, which would protect the sample surface (sec. 3.2).

\subsection{Substrate charging effects}

There has been an attempt to explain the electric property changes by irradiation-induced charging of the substrate (back-gate dielectric) just under the SWCNT (Marquardt et al., 2008. Vijayaraghavan et al., 2010). They observed a large conductivity decrease of onsubstrate SWCNT devices by electron irradiation in a SEM. Furthermore, they reversibly and repeatedly recovered the electric properties by applying a high bias voltage $(\sim 10 \mathrm{~V})$ to the SWCNT. The phenomena they observed seem to be essentially the same as ours. However, they ascribed the conductivity decrease to a local band gap opening caused by irradiation-induced charging of the dielectric $\mathrm{SiO}_{2}$ layer just under the SWCNT. Actually, theoretical calculations predict that a uniform (Li et al., 2003) or inhomogeneous (Rotkin \& Hess, 2004) electric field can open a gap in a metallic SWCNT of specific chiralities. Marquardt et al. (Marquardt et al., 2008) and Vijayaraghavan et al. (Vijayaraghavan et al., 2010) think that electron irradiation in a SEM causes such local and inhomogeneous charging of the dielectric. In their model, the recovery is explained by a release of trapped charges in the vicinity of the SWCNT caused by the high-bias voltage applied to the drain electrodes. In a conventional on-substrate device, a large electric field may be produced by irradiation-induced charging, considering that the field strength is inversely proportional to the square of the distance.

However, the substrate charging model does not at all explain the fact that the irradiationinduced conductivity decrease is as ever observed for suspended SWCNTs, as shown in Fig. 4. The theoretical calculations have predicted that an extraoridinarily high electric field of $\sim 1$ $\mathrm{Vnm}^{-1}$ barely opens a band gap of several ten milli-electron volts. It is very unlikely that such a high electric field is formed at SWCNTs suspended $300 \mathrm{~nm}$ above the substrate. In fact, a simulation has been performed under a condition where the gate voltage was applied from a metal tip located only $0.5 \mathrm{~nm}$ from a SWCNT (Rotkin \& Hess, 2004). Similarly, this model cannot explain the degradation of Raman and PL spectra of suspended SWCNTs (Figs. 1 and 9). Moreover, electron (hole) injection-induced band gap opening has been observed for a metallic SWCNT lying on a metal substrate, which does not have charge trap sites (Yamada et al., 2009). This model does not explain the observed band gap value, either. The calculations show that the maximum value of the field-induced band gap is at most $\sim 0.1$ $\mathrm{eV}$, which is not sufficient to explain the almost insulating properties observed at room temperature. In fact, an energy barrier of $\sim 0.6 \mathrm{eV}$ was observed for a SWCNT whose roomtemperature electric properties were converted from metallic to semiconducting by irradiation (sec. 5). Finally, it should be noted that the irradiation-induced conductivity decrease has been observed in all measured SWCNTs, whereas, in the theoretical calculations, band gaps open only in SWCNTs having certain chiralities. Considering that the irradiation-induced physical property changes can recover at a moderate temperature $\left(\sim 300{ }^{\circ} \mathrm{C}\right.$ [Fig. 7]), the high bias-induced recovery observed in refs. 7 and 8 seems to be due 
to annealing by Joule heating. I do not deny an electric field-induced band gap opening in a metallic SWNT. However, I do not think that such a high or inhomogeneous electric field is produced by simple SEM observation or line scans.

\subsection{Irradiation-induced heating effects}

Low-energy electron and photon irradiation may increase the temperature of the irradiated SWCNTs. However, the heating effect itself does not explain the low-energy irradiation damage at all because less damage is observed at higher temperatures, as shown in Fig. 1. Originally, SWCNTs are thermally very stable materials. Thus, at least under usual conditions, irradiation-induced heating itself would not damage the SWCNTs in a vacuum, if ever. In practice, damage is often observed during Raman measurements in air when the excitation laser power is too large. However, this is not low-energy irradiation damage, but instead would be combustion, because this damage is not observed in a vacuum or an inert gas atmosphere.

\section{Conclusion}

I have shown that low-energy electron and photon irradiation solely damages SWCNTs. The low-energy irradiation damage extinguishes the characteristic optical and electric properties and reduces chemical tolerance. Thus, we have to pay attention to the damage when we use analytical tools that use low-energy electrons (SEM, LEEM etc.) and VUV light or soft X-rays (photoemission spectroscopy using bright light). The defects have some unique properties. The damage and recovery are reversible, indicating that the number of carbon atoms is preserved. The damage strongly depends on diameter. That is, thinner SWCNTs are more severely damaged. The damage has been observed in SWCNTs but not in MWCNTs, suggesting that it is characteristic of low-dimensional structures or nanostructures. The activation energy of the defect healing depends on the extent of the damage and was evaluated to be about 0.7 to $1.4 \mathrm{eV}$. Because of the relatively small activation energy, the defects can be healed even at room temperature or below, and less damage occurs at higher temperatures. I also showed that the irradiation-induced defects can convert the room temperature electric properties of a metallic SWCNT to semiconducting. The conversion can be explained by the local band gap opening caused by the defect and gate-voltage-induced band bending in the metallic SWCNT. Energetically, the low-energy is still sufficiently larger than the $\mathrm{C}-\mathrm{C}$ bond energy and can therefore break the bonds. Future studies should address the detailed defect structure.

\section{Acknowledgment}

This work has been done through cooperation of many coworkers. I thank all of my coworkers for their cooperation and assistance in this work.

\section{References}

An, K. H.; Heo, J. G.; Jeon, K. G.; Bae, D. J.; Jo, C.; Yang, C. W.; Park, C. Y.; Lee, Y. H.; Lee, Y. S. \& Chung Y. S. (2002). X-ray photoemission spectroscopy study of fluorinated single-walled carbon nanotubes. Appl. Phys. Lett. Vol. 80, No. 22 (April 2002), pp. 4235-7. 
Arima, S.; Lee, S.; Mera, Y.; Ogura, S.; Fukutani, K.; Sato, S.; Tohji, J. \& Maeda, K. (2009). Electron-stimulated defect formation in single-walled carbon nanotubes studied by hydrogen thermal desorption spectroscopy. Appl. Surf. Sci. Vol. 256, No. 4 (June 2009), pp. 1196-9.

Bachtold, A.; Henny, M.; Terrier, C.; Strunk, C.; Schonenberger, C.; Salvetat, J. P.; Bonard, J. M. \& Forro, L. (1998). Contacting carbon nanotubes selectively with low-ohmic contacts for four-probe electric measurements. Appl. Phys. Lett. Vol. 73, No. 2 (July 1998), pp. 274-6.

Berthe, M.; Yoshida, S.; Ebine, Y.; Kanazawa, K.; Okada, A.; Taninaka, A.; Takeuchi, O.; Fukui, N.; Shinohara, H.; Suzuki, S.; Sumitomo, K.; Kobayashi, Y.; Grandidier, B.; Stievenard, D. \& Shigekawa, H. (2007). Reversible defect engineering of singlewalled carbon nanotubes using scanning tunneling microscopy. Nano Lett. Vol. 7, No. 12 (November 2007), pp. 3623-7.

Chen, B. H.; Wei, J. H.; Lo, P. Y.; Pei, Z. W.; Chao, T. S.; Lin, H. C. \& Huang, T. Y. (2006). Novel method of converting metallic-type carbon nanotubes to semiconductingtype carbon nanotube field-effect transistors. Jpn. J. Appl. Phys. Vol. 45, No. 4B (April 2006), pp. 3680-5.

Dresselhaus, M. S.; Dresselhaus, G.; Saito, R. \& Jorio, A. (2005). Raman spectroscopy of carbon nanotubes. Phys. Rep., Vol. 409, (2005), pp. 47-99.

Hashimoto, A.; Suenaga, K.; Gloter, A.; Urlta, K. \& Iijima, S. (2004). Direct evidence for atomic defects in graphene layers. Nature Vol. 430, No. 7002 (August 2004), pp. 870-3.

Hobara, R.; Yoshimoto, S.; Ikuno, T.; Katayama, M.; Yamauchi, N.; Wongwiriyapan, W.; Honda, S.; Matsuda, I.; Hasegawa, S. \& Oura, K. (2004). Electric transport in multiwalled carbon nanotubes contacted with patterned electrodes. Jpn. J. Appl. Phys. Vol. 43, No. 8B (July 2004), pp. L1081-4.

Kanzaki, K.; Suzuki, S.; Inokawa, H.; Ono, Y.; Vijayaraghavan, A. \& Kobayashi, Y. (2007). Mechanism of metal-semiconductor transition in electric properties of singlewalled carbon nanotubes induced by low-energy electron irradiation. J. Appl. Phys. Vol. 101, No. 3 (Feburuary 2007), pp. 034317-1-4.

Krasheninnikov, A. V.; Nordlund, K.; Lehtinen, P. O.; Foster, A.; Ayuela, S. \& Nieminen, R. M. (2004). Adsorption and migration of carbon adatoms on zigzag carbon nanotubes. Carbon Vol. 42, No. 5-6, (January 2004), pp. 1021-5.

Krasheninnikov, A. V. \& Nordlund, K. (2010). Ion and electron irradiation-induced effects in nanostructured materials. J. Appl. Phys. Vol. 107, No. 7 (April 2010), pp. 071301-1-70.

Li, Y.; Rotkin, V. R. \& Ravaioli U. (2003). Electronic response and bandstructure modulation of carbon nanotubes in a transverse electric field. Nano Lett. Vol. 3, No. 2 (November 2003), pp. 183-7.

Marquardt, C. W.; Dehm, S.; Vijayaraghavan, A.; Blatt, S.; Hennrich, F. \& Krupke, R. (2008). Reversible metal-insulator transitions in metallic single-walled carbon nanotubes. Nano Lett. Vol. 8, No. 9 (August 2008), pp. 2767-72.

Lee, S.; Kim, G.; Kim, H.; Choi, B.; Lee, J.; Jeong, B. W.; Ihm, J.; Kuk, Y. \& Kahng, S. J. (2005). Paired gap states in a semiconducting carbon nanotube: Deep and shallow levels. Phys. Rev. Lett. Vol. 95, No. 16 (October 2005), pp. 166402-1-4.

Matsumoto, K.; Kinoshita, S.; Gotoh, Y.; Kurachi, K.; Kamimura, T.; Maeda, M.; Sakamoto, K.; Kuwahara, M.; Atoda, N. \& Awano, Y. (2003). Jpn. J. Appl. Phys. Vol. 42, No. 4B (Janurary 2003), pp. 2415-8. 
Mera, Y.; Harada, Y.; Arima, S.; Hata, K.; Shin, S. \& Maeda, K. (2009). Defects generation in single-walled carbon nanotubes induced by soft x-ray illumination. Chem. Phys.Lett. Vol. 473, No. 1-3 (March 2009), pp. 138-41.

Mera, Y.; Fujikawa, T.; Ishizaki, K.; Xiang, R.; Shimomi, J.; Maruyama, S.; Kakiuchi, T.; Mase, K. \& Maeda, K. (2010). Ion desorption from single-walled carbon nanotubes induced by soft x-ray illumination. Jpn. J. Appl. Phys. Vol. 49, No. 10 (October 2010), pp. 105104-1-5.

Mizutani, T.; Ohnaka, H.; Okigawa, Y.; Kishimoto, S. \& Ohno, Y. (2009). A study of preferential growth of carbon nanotubes with semiconducting behavior grown by plasma-enhanced chemical vapor deposition. J. Appl. Phys. Vol. 106, No. 7 (October 2009), pp. 073705-1-5.

Ohnaka, H.; Kojima, Y.; Kishimoto, S.; Ohno, Y \& Mizutani, T. (2006). Fabrication of carbon nanotube field effect transistors using plasma-enhanced chemical vapor deposition grown nanotubes. Jpn. J. Appl. Phys. Vol. 45, No. 6B (June 2006), pp. 5485-9.

Ohno, Y. (2010). Environmentaleffects on photoluminescence of single-walled carbon nanotubes. In: Carbon Nanotubes, InTech, ISBN 978-953-307-054-4.

Okada, S. (2007). Energetics and electronic structures of carbon nanotubes with adatomvacancy defects. Chem. Phys. Lett. Vol. 447, No. 4-6 (September 2007), pp. 263-7.

Onoe, J.; Nakayama, T.; Aono, M. \& Hara T. (2003). Structural and electrical properties of an electron-beam-irradiated C60 film. Appl. Phys. Lett. Vol. 82, No. 4 (January 2003), pp. 595-7..

Rotkin, V. R. \& Hess, K. (2004). Possibility of a metallic field effect transistor. Appl. Phys. Lett. Vol. 84, No. 16 (February 2004), pp. 3139-41.

Suzuki, S.; Watanabe, Y.; Ogino, T.; Homma, Y.; Takagi, D.; Heun, S.; Gregoratti, L.; Barinov, A. \& Kiskinova, M. (2004a). Observation of single-walled carbon nanotubes by photoemission microscopy. Carbon, Vol. 42, No. 3, (January 2004), pp. 559-L63.

Suzuki, S.; Kanzaki, K.; Homma, Y. \& Fukuba, S. (2004b). Low-acceleration-voltage electron irradiation damage in single-walled carbon nanotubes. Jpn. J. Appl. Phys., Vol. 43, No. 8B, (July 2004), pp. L1118-L1120.

Suzuki, S.; Takagi, D.; Homma, Y.; Kobayashi, Y. (2005a). Selective removal of carbon nanotubes utilizing low-acceleration-voltage electron irradiation damage. Jpn. J. Appl. Phys. Vol. 44, No. 4 (January 2005), pp. L133-5.

Suzuki, S.; Fukuba, S.; Kanzaki, K.; Homma, Y. \& Kobayashi, Y. (2005b). Spatially selective removal of carbon nanotubes for fabricating nanotube circuits. Proceedings of 5 th IEEE Conference on Nanotechnology. Nagoya, July 2005.

Suzuki, S. \& Kobayashi, Y. (2005). Conductivity decrease in carbon nanotubes casued by low-acceleration-voltage electron irradiation. Jpn. J. Appl. Phys. Vol. 44, No. 49 (November 2005), pp. L1498-L1501.

Suzuki, S.; Maeda, F. \& Kobayashi, Y. (2006). Photon-induced damage creation in carbon nanotubes. 30th Fullerenes Nanotube General Symposium. Nagoya, January 2006.

Suzuki, S. \& Kobayashi, Y. (2006a). Diameter dependence of low-energy electron and photon irradiation damage in single-walled carbon nanotubes. Chem. Phys. Lett. Vol. 430, No. 1-3 (September 2006), pp. 370-4.

Suzuki, S. \& Kobayashi, Y. (2006b). Processing and electric property control of carbon nanotubes by low-energy electron irradiation. NTT Technical. Review Vol. 4, No. 11 (November 2006), pp. 25-30. 
Suzuki, S. \& Kobayashi, Y. (2007a). Healing of low-energy irradiation-induced defects in single-walled carbon nanotubes at room temperature. J. Phys. Chem. C Vol. 111, No. 12 (March 2007), pp. 4524-8.

Suzuki, S. \& Kobayashi, Y. (2007b). Low-energy irradiation damage in single-walled carbon nanotubes. Mater. Res. Soc. Symp. Proc. Vol. 994, San Francisco, (April 2007), pp. F04-02-1-12.

Suzuki, S. \& Kobayashi, Y. (2008). Threshold energy of low-energy irradiation damage in singlewalled carbon nanotubes. Jpn. J. Appl.Phys. Vol. 47, No. 4 (April 2008), pp. 2040-3.

Suzuki, S.; Hashimoto, J.; Ogino, T. \& Kobayashi, Y. (2008). Electric property control of carbon nanotubes by defects. Jpn. J. Appl.Phys. Vol. 47, No. 4 (April 2008), pp. 3292-5.

Suzuki, S. Yamaya, K.; Homma, Y.; Kobayashi, Y. (2010). Activation energy of healing of low-energy irradiation-induced defects in single-wall carbon nanotubes. Carbon Vol. 48, No. 11 (May 2010), pp. 3211-7.

Suzuki, S. (2011). Origin of the electric property change of a single-wall carbon nanotube caused by low-energy irradiation: defects or substrate charging? e-J. Surf. Sci. $\mathcal{E}$ Nanotechnol. Vol. 9, (March 2011), pp. 103-6.

Suzuki, S. \& Hibino, H. (2011). Characterization of doped single-wall carbon nanotubes by Raman spectroscopy. Carbon Vol. 49, No. 7 (January 2011), pp. 2264-72.

Vijayaraghavan, A.; Marquardt, C. W.; Dehm, S.; Hennrich F. \& Krupke, R. (2010). Imaging defects and junctions in single-walled carbon nanotubes by voltage-contrast scanning electron microscopy. Carbon Vol. 48, No. 2 (September 2009), pp. 494-500.

Yamada, K.; Sato, H.; Komaguchi, T.; Mera, Y. \& Maeda, K. (2009). Local opening of a large bandgap in metallic single-walled carbon nanotubes induced by tunnel injection of low-energy electrons. Appl. Phys. Lett. Vol. 94, No. 25 (June 2009), pp. 253103-1-3.

Yan, Y. H.; Chan-Park, M. B.; Zhou, Q.; Li, C. M.; Yue, C. Y. (2005). Functionalization of carbon nanotubes by argon plasma-assisted ultraviolet grafting. Appl. Phys. Lett. Vol. 87, No. 21 (November 2005), pp. 213101-1-3.

Yang, C. M.; An, K. H.; Park, J. S.; Park, K. A.; Lim, S. C.; Cho, S. H.; Lee, Y. S.; Park, W.; Park, C. Y. \& Lee. Y. H. (2006). Preferential etching of metallic single-walled carbon nanotubes with small diameter by fluorine gas. Phys. Rev. B Vol. 73, No. 7 (February 2006), pp. 075419-1-7.

Yuzvinsky, T. D.; Fennimore, A. M.; Nickelson, W.; Esquivias, C. \& Zettl, A. (2005). Precision cutting of nanotubes with a low-energy electron beam. Appl. Phys. Lett. Vol. 86, No. 5 (January 2005), pp. 053109-1-3.

Zhang, G.; Qi, P.; Wang, X.; Lu, Y.; Mann, D.; Li, X. \& Dai, H. (2006a). Hydrogenation and hydrocarbonation and etching of single-walled carbon nantoubes. J. Am. Chem. Soc. Vol. 128, No. 18 (April 2006), pp. 6026-7.

Zhang, G.; Qi, P.; Wang, X.; Lu, Y.; Li, X.; Tu R. Bangsaruntip, S.; Mann, D.; Zhang, Li. \& Dai, H. (2006b). Selective etching of metallic carbon nanotubes by gas-phase reaction. Science Vol. 314, No. 5801 (November 2006), pp. 974-7.

Zhao, Y. B.; Poirier, D. M.; Pechman, R. J. \& Weaver, J. H. (1994). Electron stimulated polymerization of solid C60. Appl. Phys. Lett. Vol.64, No. 5 (January 1994), pp. 577-9.

Zhou, S. Y.; Girit, C. O.; Scholl, A.; Jozwiak, C. J.; Siegel, D. A.; Yu, P.; Robinson, J. T.; Wang, F. \& Zettl, A. (2009). Instability of two-dimensional graphene: Breaking sp2 bonds with soft x rays. Phys. Rev. B Vol. 80, No. 12 (September 2009), pp. 121409-1-4. 


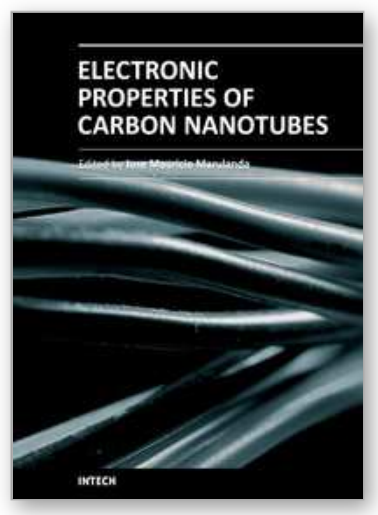

\author{
Electronic Properties of Carbon Nanotubes \\ Edited by Prof. Jose Mauricio Marulanda
}

ISBN 978-953-307-499-3

Hard cover, 680 pages

Publisher InTech

Published online 27, July, 2011

Published in print edition July, 2011

Carbon nanotubes (CNTs), discovered in 1991, have been a subject of intensive research for a wide range of applications. These one-dimensional (1D) graphene sheets rolled into a tubular form have been the target of many researchers around the world. This book concentrates on the semiconductor physics of carbon nanotubes, it brings unique insight into the phenomena encountered in the electronic structure when operating with carbon nanotubes. This book also presents to reader useful information on the fabrication and applications of these outstanding materials. The main objective of this book is to give in-depth understanding of the physics and electronic structure of carbon nanotubes. Readers of this book should have a strong background on physical electronics and semiconductor device physics. This book first discusses fabrication techniques followed by an analysis on the physical properties of carbon nanotubes, including density of states and electronic structures. Ultimately, the book pursues a significant amount of work in the industry applications of carbon nanotubes.

\title{
How to reference
}

In order to correctly reference this scholarly work, feel free to copy and paste the following:

Satoru Suzuki (2011). Low-Energy Irradiation Damage in Single-Walled Carbon Nanotubes, Electronic Properties of Carbon Nanotubes, Prof. Jose Mauricio Marulanda (Ed.), ISBN: 978-953-307-499-3, InTech, Available from: http://www.intechopen.com/books/electronic-properties-of-carbon-nanotubes/low-energyirradiation-damage-in-single-walled-carbon-nanotubes

\section{INTECH}

open science | open minds

\section{InTech Europe}

University Campus STeP Ri

Slavka Krautzeka 83/A

51000 Rijeka, Croatia

Phone: +385 (51) 770447

Fax: +385 (51) 686166

www.intechopen.com

\section{InTech China}

Unit 405, Office Block, Hotel Equatorial Shanghai

No.65, Yan An Road (West), Shanghai, 200040, China

中国上海市延安西路 65 号上海国际贵都大饭店办公楼 405 单元

Phone: +86-21-62489820

Fax: $+86-21-62489821$ 
(C) 2011 The Author(s). Licensee IntechOpen. This chapter is distributed under the terms of the Creative Commons Attribution-NonCommercialShareAlike-3.0 License, which permits use, distribution and reproduction for non-commercial purposes, provided the original is properly cited and derivative works building on this content are distributed under the same license. 